\section{Phlebitis predictors in cardiac patients undergoing peripheral intravenous therapy}

\section{Abstract}

Objective: To determine the incidence of phlebitis and its predictive factors in cardiac patients hospitalized in a coronary care unit.

Method: this study is a prospective cohort, conducted with 60 adult cardiac patients using peripheral intravenous therapy, admitted to the hospital sector of the coronary unit from August to December 2012. The data were analyzed using BioEstat programs 5.0 and SPSS 20.0. Non-parametric data were analyzed using the chi-square test, Fisher exact test, and Mann-Whitney test. A p-value $<0.05$ was considered statistically significant.

Results: The incidence of phlebitis was $12 \%$ about the proportionality of peripheral venous catheters $(n=139)$. There were four variables identified as predictive factors for phlebitis, diabetes mellitus and dyslipidemia $(p=0.02)$, hospitalization for three days and the number punches were over two $(p<0.001)$.

Conclusion: In this study, the phlebitis rate was twice greater than the established by the international standards. The cardiac patients with diabetes mellitus and dyslipidemia were 2.7 and 2.8 times the risk for phlebitis development than those who did not have these factors, respectively.
Adriana Correia de Lima', Rivaldo Venâncio da Cunha1, Marisa Dias Rolan Loureiro', Maria Lúcia Ivo', Albert Schiaveto de Souza1, Elenir Rose Jardim Curi Pontes ${ }^{1}$, Aston Marques da Silva Junior ${ }^{1}$, Isabelle Campos de Azevedo ${ }^{2}$, Marcos Antonio Ferreira Júnior ${ }^{2}$

1 Federal University of Mato Grosso do Sul, Campo Grande/MS, Brazil.

2 Federal University of Rio Grande do Norte, Natal/RN, Brazil.

Contact information:

\section{Marcos Antonio Ferreira Júnior.}

Đmarcos_nurse@hotmail.com

\section{Keywords}

Phlebitis; latrogenic Disease; Patient Safety; Heart Diseases; Intravenous Infusions. 


\section{Introduction}

Cardiovascular diseases are circulatory system diseases with a broad spectrum of clinical syndromes and have an important impact on morbidity and mortality and hospitalization costs and outpatient monitoring [1].

According to the American Heart Association, more than 71 million Americans have one or more types of cardiovascular disease, frequently having coronary artery disease, heart failure, stroke and congenital cardiovascular defects [2]. Thus, the number of patients hospitalized with heart disease is increasing worldwide.

Peripheral intravenous therapy has a set of knowledge and techniques that range from the administration of solutions and medications in the circulatory system to the catheters care (maintenance, salinization, cover exchange, and discard) [3].

Currently, the insertion of peripheral venous catheters (PVC) is one of the most commonly invasive procedures performed in hospitalized patients [4], and may result in adverse events or traumas located around the puncture site as hematomas, phlebitis, thrombophlebitis, infiltrations, extravasation and local infection [5, 6]. Adverse events are incidents that result in unintentional damage from the care and not related to the natural evolution of the patient's disease [7].

Phlebitis is an adverse event that compromises the safety of the patient, defined as an inflammation of the vein which can be accompanied by pain, erythema, swelling and formation of palpable cord [8] and $2.5 \%$ to $70 \%$ among all patients who undergo this procedure have implications $[9,10]$.

The potential danger for patients undergoing these procedures may result in a physical, social and financial burden to the health institutions, which requires constant prevention measures for patient safety.

Thus, this study was developed to verify the incidence of phlebitis and its predictive factors in cardiac patients hospitalized in a coronary care unit.

\section{Method}

A quantitative study of epidemiological approach with a prospective cohort, conducted with adult cardiac patients admitted to the coronary unit in a hospital in the Midwest region of Brazil from August to December 2012.

For data collection, an instrument specifically designed for this study was used, which contained the data of variables: a) demographic: age, gender, and color; B) Clinical: medical diagnosis, comorbidities, length of stay in days, percutaneous coronary intervention, use of antimicrobial drugs, corticosteroids, anticoagulant and antiplatelet agent; C) characterization of venipuncture: number of punctures, type and caliber of the catheter, anatomical location and the catheter dwell time; and D) therapy interruption reasons - outcome: hospital and/or sector discharge, recommended length of stay (up to 72 hours by the institution) and local complications.

The variables showing local complications, defined as any undesirable event that had prevented the continuity of therapy were classified as pain, heat, redness, palpable cord formation, infiltration, phlebitis (scales proposed by the Infusion Nurses Society [8], hematoma, ecchymosis, obstruction and accidental deletion.

Patients with heart disease, $\geq 18$ years old, in early peripheral intravenous therapy, and those with central venous access were excluded and transferred to another sector.

The selected cardiac patients after admission in the sector were followed from the preview site inspection to the insertion and the time of the first peripheral venipuncture when the sites were observed every 24 hours until the removal of the catheter. The type of coverage and fixation was observed every day, performing the palpation at the insertion site 
and when necessary, a simple magnifying glass was used to help in the investigation of inflammatory signs. Such procedures have identified the presence of clinical manifestations and risk factors as well as the causes of local complications. Hospital records of all patients investigated for evaluation of additional data were consulted, such as the occurrence of adverse events, infection revealed by positive blood culture and intravenous drug prescription.

Each study subject was observed throughout the treatment when the presence of an event did not make impossible the occurrence of another of the same type or different. Thus, it was characterizing the study discontinuity.

Data were organized in tables with absolute and relative frequencies, and their variables were analyzed using BioEstat 5.0 and SPSS 20.0 programs. Non-parametric data were analyzed using the chisquare test, Fisher exact test, and Mann-Whitney test. A p-value $<0.05$ was considered statistically significant.

The research study protocol was approved in its ethical, and methodological aspects by the Ethics Committee of the Federal University of Mato Grosso do Sul (Protocol 2242 and CAAE 0342.0.049.00111). Participants were informed about the objectives and procedures of the study and signed the Informed Consent Form before their inclusion in the study.

\section{Results}

The study included 60 patients, 32 of them $(53.3 \%)$ were male and 28 were female, with an average age of 64 years old (SD \pm 12.69$)$. Most participants (38) were more than 60 years old, 25 (41.7\%) were between 60-75 years old, 13 (21.6\%) were over 76 years old, with a predominance of white in 50 participants (83 3\%) among all subjects studied.

The predominant clinical variables were the acute coronary syndromes in 19 patients (31.7\%), while
12 (20\%) had stable angina and seven (11.7\%) had acute myocardial infarction. Fifteen patients (24.9\%) had coronary artery disease. Cardiac arrhythmias were observed in 16 patients, (26.6\%) and the most common were the acute atrial fibrillation in 10 subjects (16.6\%). The most common comorbidities were hypertension in 53 patients (88.3\%), diabetes mellitus in 28 patients (46.7\%), dyslipidemia in 31 of them (51.6\%) and smoking in 12 participants (20\%).

At the end of the follow-up, there were 17 patients analyzed who had phlebitis and 43 without it.

Table 1 shows that gender, age, ethnicity, and comorbidities of hypertension and smoking were not statistically significant for the development of phlebitis. However, there was a significant association between diabetes mellitus and dyslipidemia.

Twenty-three patients (38.3\%) underwent Percutaneous Coronary Intervention; that is the method used for coronary reperfusion. Six patients (9.9\%) used antimicrobial and one patient (1.7\%) used corticosteroids. The combined platelet therapy and antiplatelet anticoagulant were used in 50 (83.3\%) patients.

Other types of drugs or solutions used did not show relevance in the occurrence of phlebitis. In 60 patients, there were 139 peripheral punctures successfully performed, 55 of them (91.7\%) were urgent, coming from the emergency unit of chest pain. The average of inserted catheter was 2.3 per patient, 14 of them (23.3\%) were punctured once, $46(76.7 \%)$ were subjected to two punches, 19 (31.7\%) had three punches, 10 (16.7\%) had four, three had five punctures, and one patient had six peripheral punctures. In all inserts, the intravenous device used was the type "off-the-needle", with polyurethane (Vialon®). Catheters caliber 20 were used in 105 punctures (75.5\%) and caliber 22 in other 20 (14.4\%). The sites chosen for the puncture were predominantly the forearm with 69 cases $(49.7 \%)$, followed by the back of the hand with 39 (28.0\%) and antecubital fossa with 31 (22.3\%) cases. 
Table 1. Clinical and demographic characteristics of cardiac patients using intravenous therapy in coronary care unit being monitored to verify phlebitis. Campo Grande, MS. Brazil $2012(n=60)$.

\begin{tabular}{|c|c|c|c|c|c|c|}
\hline Variables & $\begin{array}{l}\text { Present } \\
\text { Phlebitis }\end{array}$ & $\begin{array}{l}\text { Absent } \\
\text { Phlebitis }\end{array}$ & Total & $\mathbf{p}^{*}$ & $\mathbf{R R}^{* *}$ & $\mathrm{Cl} 95 \% * * *$ \\
\hline Gender & & & & 0.267 & 1.43 & $0.66-3.11$ \\
\hline Female & 10 & 18 & 28 & & & \\
\hline Male & 7 & 25 & 32 & & & \\
\hline Total & 17 & 43 & 60 & & & \\
\hline Age (years) & & & & 0.151 & 0.53 & $0.20-1.43$ \\
\hline Up to 60 & 4 & 18 & 22 & & & \\
\hline More than 60 & 13 & 25 & 38 & & & \\
\hline Total & 17 & 43 & 60 & & & \\
\hline Skin color & & & & 0.152 & 3.20 & $0.48-21.46$ \\
\hline White & 16 & 34 & 50 & & & \\
\hline Non-white & 1 & 9 & 10 & & & \\
\hline Total & 17 & 43 & 60 & & & \\
\hline \multicolumn{7}{|c|}{ Associated comorbidities } \\
\hline $\mathrm{SAH}$ & & & & 0.333 & 2.11 & $0.33-13.58$ \\
\hline Present & 16 & 37 & 53 & & & \\
\hline Absent & 1 & 6 & 7 & & & \\
\hline Total & 17 & 43 & 60 & & & \\
\hline Diabetes mellitus & & & & 0.020 & 2.74 & $1.10-6.83$ \\
\hline Present & 12 & 16 & 28 & & & \\
\hline Absent & 5 & 27 & 32 & & & \\
\hline Total & 17 & 43 & 60 & & & \\
\hline Dyslipidemia & & & & 0.029 & 2.81 & $1.02-7.72$ \\
\hline Present & 12 & 19 & 31 & & & \\
\hline Absent & 4 & 25 & 29 & & & \\
\hline Total & 16 & 44 & 60 & & & \\
\hline Smoking & & & & 0.259 & 0.53 & $0.14-2.02$ \\
\hline Present & 2 & 10 & 12 & & & \\
\hline Absent & 15 & 33 & 48 & & & \\
\hline Total & 17 & 43 & 60 & & & \\
\hline
\end{tabular}

* Fisher exact test; $* *$ Relative Risk; ${ }^{* * *}$ Confidence Interval.

As for the length of time, the use of a device exceeded to 72 hours. In 50 cases (35.9\%) the device was maintained for up to 24 hours; in cases $42(30.2 \%)$ it remained between 24 and 48 hours, and in 46 cases (33.0\%) between 48 and 72 hours.

The main reasons for removal of the 139 peripheral venous catheters (PVC) were patient discharge in 55
(39.6\%) cases, followed by removal of a length of stay in 30 cases $(21.6 \%)$ due to the protocol used by the institution, changing them every 72 hours.

Of the 60 patients monitored, 34 (56.7\%) developed local complications. However, the phlebitis was observed in 17 (28.3\%) patients.

Regarding the 139 PVC entered, 54 of them 
(38.8\%) developed complications, and 17 of them $(12.2 \%)$ were phlebitis, ten $(58.8 \%)$ in female and seven $(41.2 \%)$ of the male. There was mainly grade 2 phlebitides in seven cases, grade 3 in seven patients and grade 1 in three patients.

The catheters permanence time in cases of phlebitis was 48 hours in 10 cases (58.8\%), other 5 (29.4\%) cases there was 24 hours and in two cases there were 72 hours of permanence.

Events such as heat, redness and pain were also analyzed individually as complications of peripheral intravenous therapy. Although alone they meant reasons for discontinuation of therapy, they were an important factor in the overall analysis.

In all cases of complications related to PVC, the average length of hospital stay was significantly higher than on patients who did not have complications. There was a higher incidence of complications such as pain, heat, redness, palpable cord formation and obstruction in patients with more than three days of hospitalization (Table 2).

It was observed that the number of hospitalization days for patients who developed complications related to phlebitis was higher $(6.47 \pm 3.58)$ than in those without this occurrence (3.58 \pm 1.24$)$ (Figure 1).

Figure 1: Box plot chart with analysis of days in intravenous therapy and phlebitis outcome $(n=60)$. Horizontal line: average, Box: interquartile interval, Bar: variation range, Symbol: outlier.

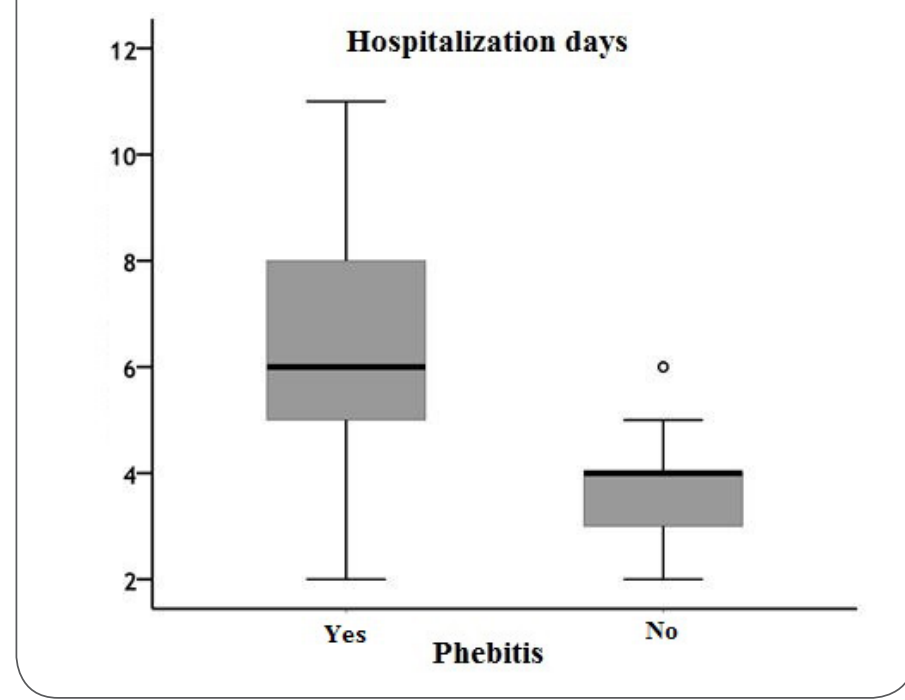

Table 1. Average of hospitalization days in cardiac patients using peripheral intravenous therapy according to the occurrence of local complications. Campo Grande, MS, Brazil, in 2012 ( $n=60)$.

\begin{tabular}{|c|c|c|c|c|c|}
\hline Local complications & $\mathbf{N}$ & $\begin{array}{c}\text { Average of } \\
\text { days }\end{array}$ & SD* & $\mathrm{T} * *$ & $P * * *$ \\
\hline Pain & & & & 14.28 & $<0.001$ \\
\hline Yes & 26 & 5.50 & 2.57 & & \\
\hline No & 34 & 3.59 & 1.28 & & \\
\hline Heat & & & & 33.64 & $<0.001$ \\
\hline Yes & 17 & 6.47 & 2.60 & & \\
\hline No & 43 & 3.61 & 1.24 & & \\
\hline Redness & & & & 40.27 & $<0.001$ \\
\hline Yes & 19 & 6.42 & 2.46 & & \\
\hline No & 41 & 3.49 & 1.14 & & \\
\hline Palpable cord formation & & & & 40.35 & $<0.001$ \\
\hline Yes & 9 & 7.67 & 2.60 & & \\
\hline No & 51 & 3.84 & 1.46 & & \\
\hline
\end{tabular}

*Standard deviation; ** Averages Differences test; ${ }^{* * *}$ Mann-Whitney Test. 
The average number of punctures among patients with phlebitis $(3.71 \pm 1.05)$ were significantly greater than those patients without phlebitis $(1.77 \pm 0.61)$ (Figure 2).

Figure 2: Box plot Chart with analysis of the number of punctures and phlebitis outcome $(n=60)$. Horizontal line: Average, Box: interquartile interval, Bar: variation range, Symbol: outlier. * Statistically significant difference compared to patients without phlebitis (Mann-Whitney test, $\mathrm{p}<0.001$ ).

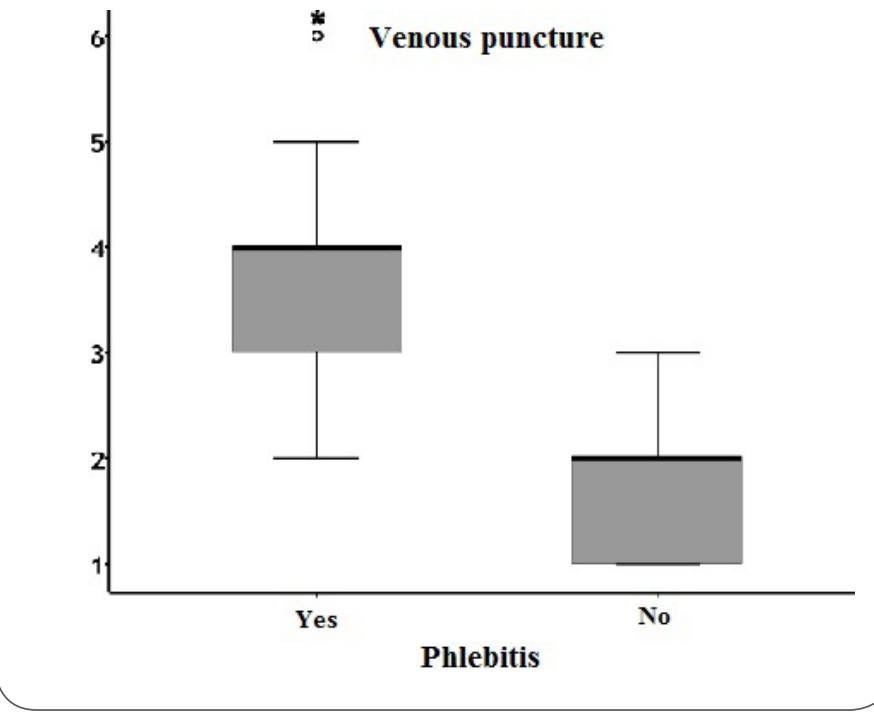

There were no isolated or associated complications among patients such as local infection, bloodstream infection, tremors, chills, secretion, and fever.

\section{Discussion}

This cohort study observed 60 hospitalized cardiac patients in a coronary care unit, with the insertion of a total of 139 peripheral venous catheters, an incidence of $33 \%$ complications found, with the occurrence of phlebitis in 17 (12.2\%) insertion sites. Another cohort study conducted in southeastern
Brazil, with 60 neurosurgical patients (152 PVC) found a percentage of $10.5 \%$ of phlebitis [11], similar to this study. Two other cohort studies in hospitalized adults found phlebitis rates of $25.8 \%$ in 76 patients with cardiorespiratory and renal disease (155 PVC) [12] and 60\% in 100 patients with pulmonary disease and cardiac patients (234 PVC), respectively [13].

In Germany, a prospective cohort study found in 1,582 patients (2,495 PVC), average rates of phlebitis of $27 \%$ [14]. Another prospective observational study in Portugal with older adults (average age of 75.92 years old, $S D=14.52$ ) showed that in 1,244 catheters, the incidence of phlebitis was $11.09 \%$ [15].

The phlebitis incidence rates are different in the studies analyzed. It is noteworthy that when comparing these rates in national and international literature, it is necessary a careful analysis due to the uniqueness of the population, the method, and the sampling process, the materials used and the established protocols, including those relating to the standardization of terms.

The variable gender was not significant for the occurrence of phlebitis in the studied sample. However, studies have shown influence both in female [16-17] as male [18].

The skin color of the individuals as the studies carried out in other Brazilian studies showed no relationship with episodes of phlebitis found $[9,19]$.

In this study, the average age was $64.44 \pm 12.57$ years old, and $63.33 \%$ were over 60 years old. Advanced age is considered an isolated risk factor for many diseases and for determining longer hospital stays and a higher incidence of adverse reactions to different drugs and procedures [20].

The age was not significant in the development of phlebitis in the patients with more or less than 60 years old. Different results with 100 elderly patients (72.0 \pm 7.7 years old) were found in Campinas, SP, demonstrating that they are susceptible to the occurrence of iatrogenic events such as infiltration, 
obstruction and phlebitis in peripheral venous access before 72 hours of puncture [21].

The predominant clinical variables in this study were acute coronary syndromes and among the most frequent comorbidities, diabetes mellitus and dyslipidemia were the significant factors for phlebitis.

Diabetes mellitus and dyslipidemia are firmly established risk factors for atherosclerosis, which is a complex chronic inflammatory process that affects elastic and muscular arteries. While the blood circulation has been extensively studied, there is little known about the pathophysiology of venous diseases related to these two diseases and also hypertension [22], present in $88.3 \%$ of patients.

A higher occurrence of inflammatory signs in patients with Diabetes and dyslipidemia was found. This fact may be related to the comorbidity presented by this group of patients and the various factors that lead to a predisposition to injury in the vascular endothelium. Diabetes mellitus has also been cited as a relevant factor for the development of phlebitis in another study [19].

The average of more than three days in hospitalization favored the development of phlebitis and other complications. These results are corroborated in a retrospective study of 100 patients' records when there was a statistically significant difference in length of stay between cases with reported iatrogenic (14.0 018.3 days) compared to those without it $(7.1 \pm 9.5$ days $)$. Infiltration, obstruction and phlebitis in peripheral venous access were among the most frequent iatrogenic [21].

Statistically significant association in patients who were hospitalized for more than eighteen days and higher frequency of phlebitis was also found in a study of 100 patients in the Brazilian Federal District [13]. A cohort of 380 patients showed a strong correlation of the patient's length of stay in intensive care units with the number of adverse events ( $p$ $<0.0001)$, including phlebitis. In the adverse events associated with Nursing performance, phlebitis corresponded to $11.58 \%$ of these incidents [23]. Although there are limited descriptions of the length of stay as a predisposing factor for phlebitis $[13,21$, 23], this study could address this variable.

There were 23 patients (38.3\%) of 60 in this study who underwent percutaneous coronary intervention and 50 (83.3\%) used adjunctive pharmacological therapy using antiplatelet and oral, intravenous or subcutaneous anticoagulants. The administration of a dual antiplatelet therapy (aspirin + derived thienopyridine) is indicated for the reduction of thrombosis in patients with coronary artery disease with pharmacological stent implantation, and its maintenance is recommended for a minimum period of one year. [24]

Some of the patients using this therapy had undergone stent implantation previously in other previous hospitalizations. In both cases, adjunctive therapy was used.

In nine cases, the patients had bruises during the observation period without interruption of therapy. In only one case, the presence of hematoma was interrupted. The increased number of patients with bruises may be due to oral antiplatelet therapy and/ or intravenous and oral anticoagulation therapy and/ or subcutaneous implemented as adjunctive therapy in cases of patients with stent implantation.

Patients using these drugs may have the potential for complications such as bleeding at the peripheral puncture site. In such cases, it is recommended the daily monitoring of the integumentary system for early detection of signals such as petechia, bruises, and hematomas.

There was a significant association between the higher number of punctures per patient with the occurrence of phlebitis, similar to other studies [9, 13]. In 17 patients who developed phlebitis, 15 of them $(87 \%)$ underwent more than two punctures.

There are reports of complications for the length of stay, type, catheter caliber and device location. However, these variables did not show significant 
impact as predictors for the occurrence of phlebitis or complications in this study. It should be noted that the time of 72 hours stay was strictly fulfilled by the institution, the polyurethane (Vialon $®)$ catheter was used in $100 \%$ of patients, having a lower incidence thrombogenic, more flexible, a lower risk of phlebitis [6, 25] and more 20 and 22 caliver have been used as recommended in the literature, due to the fact of being less traumatic $[6,17]$.

\section{Conclusion}

In this study, adults with heart disease had a twice higher rate for development of phlebitis than the $5 \%$ established for patients by international standards in general of the Infusion Nursing Society.

The findings were about the cardiac patients with diabetes and dyslipidemia with a higher relative risk of 2.7 and 2.8 times, respectively for the development of phlebitis compared to those who did not have these factors.

Therefore, the cardiac patients using peripheral intravenous therapy showed three days in the hospital and more than two punctures as predictors of phlebitis.

Occurrences above the established standards interfere with the patient's level of security, becoming susceptible to iatrogenic complications in the hospital. Even Although unintentional damaging effects, the relevance of this study is to encourage their reduction through the production of important information to the development planning activities for safer care to cardiac patients undergoing this therapy.

\section{References}

1. Serrano Júnior CV, Timerman A, Stefanini E. Tratado de cardiologia SOCESP. 2. Ed. Barueri (SP): Manole; 2009. v. 1, p. 7-16.

2. Smeltzer SC, Bare BG. Brunner \& Suddarth: tratado de enfermagem médico-cirúrgica. 10. Ed. Rio de Janeiro (RJ): Guanabara Koogan; 2009. 5.573 p.

3. Machado AF, Pedreira MLG, Chaud MN. Prospective, randomized and controlled trial on the dwell time of peripheral intravenous catheters in children, according to three dressing regimens. Rev. Latino-Am. Enfermagem. 2005; 13(3): 291-8. Available from: http://www.scielo.br/pdf/rlae/v13n3/v13n3a02.pdf

4. Lopez V, Molassiotis A, Chan WK, Ng F, Wong E. An intervention study to evaluate nursing management of peripheral intravascular devices. J Infus Nurs. 2004; 27(5): 322-31. Available from: http:// www.ncbi.nlm.nih.gov/pubmed/15385896

5. O'Grady NP, Alexander M, Burns LA, Dellinger EP, Garland J, Heard SO, et al. 2011 Guidelines for the prevention of intravascular catheter-related infections [internet]. Atlanta (GA): Centers for Disease Control and Prevention; 2011. 83p. Available from: http://www.cdc.gov/hicpac/pdf/guidelines/bsiguidelines-2011.pdf

6. Phillips LD. Manual de terapia intravenosa. 4. Ed. Porto Alegre (RS): Artmed; 2005. 551p.

7. Organização Mundial de Saúde (OMS). Estrutura concetual da classificação internacional sobre segurança do doente: relatório técnico final [internet]. Lisboa (Pt): OMS; 2011. 145p. Available from: https://www.dgs.pt/documentos-e-publicacoes/ classificacao-internacional-sobre-seguranca-do-doente-png. aspx

8. Infusion Nurses Society. Infusion nursing standards of practice. J Infus Nurs. 2006; 29(1 Suppl): S1-92.

9. Uslusoy E, Mete S. Predisposing factors to phlebitis in patients with peripheral intravenous catheters: a descriptive study. J Am Acad Nurse Pract. 2008; 20(4): 172-80.

10. Martínez JA, Piazuelo M, Almela M, Blecua P, Gallardo R, Rodríguez $S$, et al. Evaluation of add-on devices for the prevention of phlebitis and other complications associated with the use of peripheral catheters in hospitalized adults: A randomized controlled study. J Hosp Infect. 2009; 73(2): 135-42. Available from: http://www.ncbi.nlm.nih.gov/pubmed/19712998

11. Ferreira LR, Pedreira MLG, Diccini S. Phlebitis among neurosurgical patients. Acta Paul Enferm. 2007; 20(1): 30-6. Available from: http://www.scielo.br/pdf/ape/v20n1/a06v20n1. pdf

12. Magerote NP, Lima MHM, Silva JB, Correia MDL, Secoli SR. Relation between phlebitis and peripheral intravenous catheter remov. Texto Contexto Enferm. 2011; 20(3): 486-92. Available from: http://www.scielo.br/pdf/tce/v20n3/09.pdf

13. Abdul-Hak CK, Barros ÂF. The incidence of phlebitis in a medical clinical unit. Texto Contexto Enferm. 2014; 23(3): 633-8. Available from: http://www.scielo.br/pdf/tce/v23n3/0104-0707tce-2014000900013.pdf

14. Grüne F, Schrappe M, Basten J, Wenchel HM, Tual E, Stützer $\mathrm{H}$, et al. Phlebitis rate and time kinetics of short peripheral intravenous catheters. Infection. 2004; 32(1): 30-2.

15. Oliveira ASS, Parreira PMSD. Nursing interventions and peripheral 
venous catheter-related phlebitis. Systematic literature review. Rev Enf Ref. 2010; 3(2): 137-47. Available from: http://www. scielo.mec.pt/pdf/ref/vserllln2/serllln2a15.pdf

16. White SA. Peripheral intravenous therapy-related phlebitis rates in an adult population. J Intraven Nurs. 2001; 24(1): 19-24.

17. Maki DG, Ringer M. Risk factors for infusion-related phlebitis with small peripheral venous catheters. Ann Intern Med. 1991; 114(10): 845-54.

18. Cicolini G, Bonghi AP, Di Labio L, Di Mascio R. Position of peripheral venous cannulae and the incidence of thrombophlebitis: An observational study. J Adv Nurs. 2009; 65(6): 1268-73.

19. Rego Furtado LC. Incidence and predisposing factors of phlebitis in a surgery department. Br J Nurs. 2011; 20(14): S16-25.

20. Margarido NF. Complicações da cirurgia do paciente idoso. In: Colégio Brasileiro de Cirurgiões. Programa de Auto-Avaliação em Cirurgia: paciente idoso. Rio de Janeiro (RJ): Diagraphic; 2002. f. 11. p. 5-15.
21. Santos JC, Ceolim MF. latrogenias de enfermagem em pacientes idosos hospitalizados. Rev Esc Enferm USP. 2009; 43(4): 810-7.

22. Levy BI, Ambrosio G, Pries AR, Struijker-Boudier $H A$. Microcirculation in hypertension: a new target for treatment? Circulation. 2001; 104(6): 735-40.

23. Novaretti MC, Santos EV, Quitério LM, Daud-Gallotti RM. Sobrecarga de trabalho da Enfermagem e incidentes e eventos adversos em pacientes internados em UTI. Rev Bras Enferm. 2014; 67(5): 692-9.

24. Mangione JA, Mauro MFZ, Cristovão SAB. Suporte farmacológico na intervenção coronária percutânea. In: Serrano CV Jr, Timerman A, Stefanini E. Tratado de cardiologia SOCESP. 2. Ed. Barueri (SP): Manole; 2009. v. 1, p. 967-83.

25. Tagalakis V, Kahn SR, Libman M, Blostein M. The epidemiology of peripheral vein infusion thrombophlebitis: a critical review. Am J Med. 2002; 113(2): 146-51.
Publish in International Archives of Medicine

International Archives of Medicine is an open access journal publishing articles encompassing all aspects of medical science and clinical practice. IAM is considered a megajournal with independent sections on all areas of medicine. IAM is a really international journal with authors and board members from all around the world. The journal is widely indexed and classified Q1 in category Medicine. 